\title{
MMP-1 drives immunopathology in human tuberculosis and transgenic mice
}

\author{
Paul Elkington, ${ }^{1}$ Takayuki Shiomi, ${ }^{2}$ Ronan Breen, ${ }^{3}$ Robert K. Nuttall,, ${ }^{4}$ Cesar Augusto Ugarte-Gil, ${ }^{1}$ \\ Naomi F. Walker, ${ }^{1}$ Luísa Saraiva, ${ }^{1}$ Bernadette Pedersen, ${ }^{1}$ Francesco Mauri, ${ }^{5}$ Marc Lipman, ${ }^{3}$ \\ Dylan R. Edwards, ${ }^{4}$ Brian D. Robertson, ${ }^{6}$ Jeanine D'Armiento, ${ }^{2}$ and Jon S. Friedland ${ }^{1}$
}

\begin{abstract}
${ }^{1}$ Department of Infectious Diseases and Immunity, Imperial College London, London, United Kingdom. 2Department of Medicine, Columbia University, New York, New York, USA. ${ }^{3}$ Royal Free Hospital, London, United Kingdom. ${ }^{4}$ School of Biological Sciences, University of East Anglia, Norwich, United Kingdom. ${ }^{5}$ Centre for Pathology and ${ }^{6}$ Section of Microbiology, Imperial College London, London, United Kingdom.
\end{abstract}

\begin{abstract}
Mycobacterium tuberculosis can cause lung tissue damage to spread, but the mechanisms driving this immunopathology are poorly understood. The breakdown of lung matrix involves MMPs, which have a unique ability to degrade fibrillar collagens at neutral $\mathrm{pH}$. To determine whether MMPs play a role in the immunopathology of tuberculosis (TB), we profiled MMPs and their inhibitors, the tissue inhibitor of metalloproteinases (TIMPs), in sputum and bronchoalveolar lavage fluid from patients with TB and symptomatic controls. MMP-1 concentrations were significantly increased in both HIV-negative and HIV-positive patients with TB, while TIMP concentrations were lower in HIV-negative TB patients. In primary human monocytes, $M$. tuberculosis infection selectively upregulated MMP1 gene expression and secretion, and Ro32-3555, a specific MMP inhibitor, suppressed M. tuberculosis-driven MMP-1 activity. Since the mouse MMP-1 ortholog is not expressed in the lung and mice infected with $M$. tuberculosis do not develop tissue destruction equivalent to humans, we infected transgenic mice expressing human MMP-1 with M. tuberculosis to investigate whether MMP-1 caused lung immunopathology. In the MMP-1 transgenic mice, M. tuberculosis infection increased MMP-1 expression, resulting in alveolar destruction in lung granulomas and significantly greater collagen breakdown. In summary, MMP-1 may drive tissue destruction in $\mathrm{TB}$ and represents a therapeutic target to limit immunopathology.
\end{abstract}

\section{Introduction}

Mycobacterium tuberculosis infects one-third of the world's population (1) and is transmitted by the aerosol route. Although the mechanisms whereby $M$. tuberculosis evades the host immune response are increasingly well understood (2), those by which M. tuberculosis engages the immune response to drive tissue destruction and hence transmission are relatively poorly characterized (3). The events underlying this immunopathology are not well defined, in part because the mouse, one of the most useful models in which to study M. tuberculosis immunology, does not develop lung pathology similar to that of humans $(4,5)$. In humans, M. tuberculosis subverts the host immune response to drive proteolytic destruction of the extracellular matrix scaffold. The current paradigm of tuberculosis (TB) pathology proposes that caseation leads directly to cavitation $(2,4,6)$. However, this model overlooks that fact that destruction of lung extracellular matrix must be driven by proteases. Fibrillar collagens provide the lung's tensile strength and are highly resistant to enzymatic degradation $(7,8)$. Only collagenolytic MMPs can cleave these helical collagens at neutral $\mathrm{pH}(9)$.

MMPs are a family of zinc-dependent proteases that can collectively degrade all components of the extracellular matrix (8). MMP activity is tightly regulated at the level of transcription and activation by proteolytic cleavage. MMPs are specifically inhibited by tissue inhibitor of metalloproteinases (TIMPs) (9). Excessive MMP activity is implicated in diverse pulmonary pathologies characterized by extracellular matrix destruction (8). However, despite the potentially key role of MMPs in lung matrix destruction in human TB, the central mechanisms resulting in tissue damage have not been defined.

Conflict of interest: The authors have declared that no conflict of interest exists. Citation for this article: J Clin Invest. 2011;121(5):1827-1833. doi:10.1172/JCI45666.

\section{Results}

MMP-1 is increased in patients with TB. First, we profiled MMP concentrations in induced sputum and bronchoalveolar lavage fluid (BALF) from patients with respiratory symptoms being investigated for TB who had alternative final diagnoses (referred to herein as respiratory symptomatics) (demographic data in Supplemental Table 1; supplemental material available online with this article; doi:10.1172/ JCI45666DS1; diagnoses of control patients in Supplemental Table 2). Median MMP-1 and MMP-3 (stromelysin-1) concentrations normalized to total protein were significantly upregulated in patients with TB (Figure 1A and Supplemental Table 3). MMP-1 was increased by 15.6 fold $(P=0.0001)$, and MMP-3 was increased by 3.6 fold ( $P=0.0007$ by Mann-Whitney U test). MMP-8 (neutrophil collagenase) was unchanged, and MMP-13 (collagenase-3) was undetectable, demonstrating that MMP-1 is the principal secreted collagenase upregulated in TB. Concurrently, median levels of the MMP inhibitors TIMP-1 and TIMP-2 were significantly lower in patients with TB (Figure 1B; TIMP-1, 4.7-fold lower, $P=0.0418$, and TIMP-2, 138 -fold lower, $P=0.0053$ ). No difference in the concentrations of TNF- $\alpha$, IFN- $\gamma$, and IL-1 $\beta$, key cytokines in the immune response to TB, was demonstrated (Supplemental Figure 1). When analysis was performed for each clinical specimen subgroup, differences in the MMP-1 levels between TB and control patients remained significant despite the small sample size (Supplemental Table 4).

To determine whether elevated MMP-1 was a generalized phenomenon in TB, we next analyzed respiratory secretions of patients coinfected with HIV (14 controls vs. 20 patients with TB). Consistent with the first patient cohort, median MMP-1 concentrations were 5.7-fold higher in patients with TB compared with those in respiratory symptomatics (Figure $2 \mathrm{~A} ; P=0.0026)$. Analysis of induced sputum samples by casein zymography revealed 

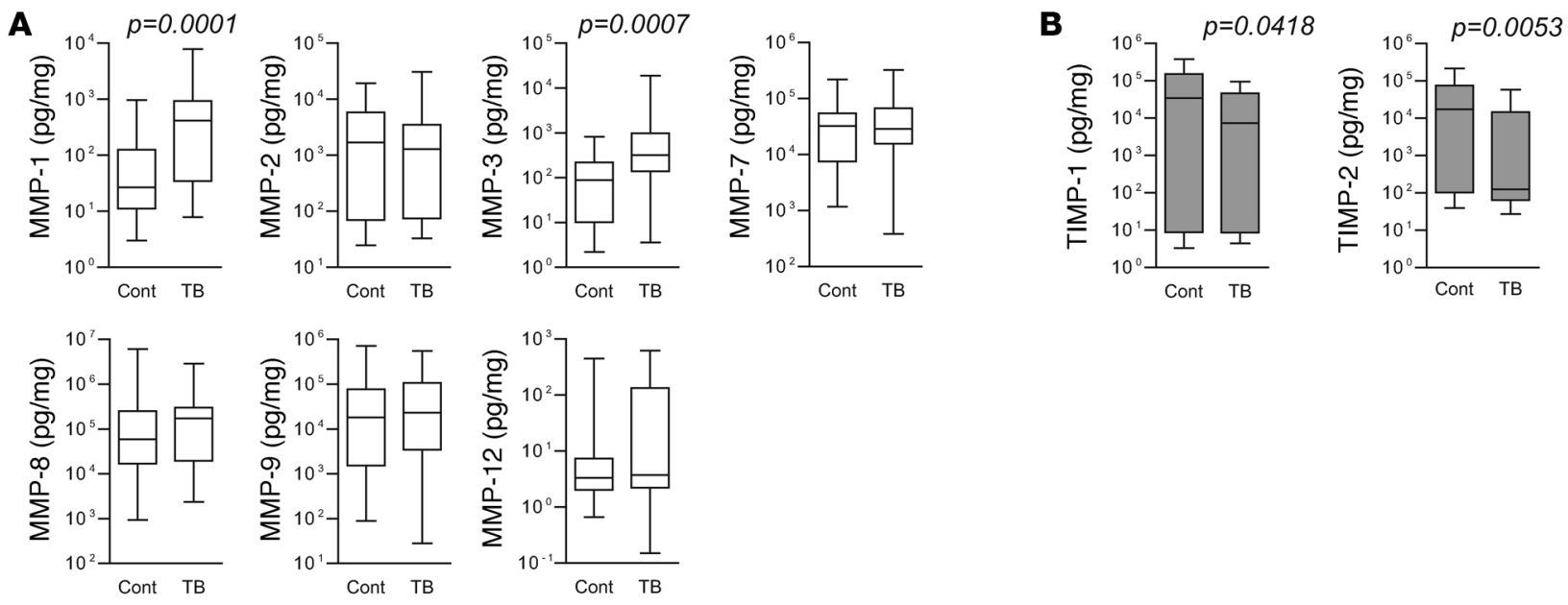

\section{Figure 1}

MMP-1 concentrations are increased in the lungs of patients with TB compared with those of symptomatic controls. Induced sputum and bronchoalveolar lavage samples were analyzed for MMP and TIMP concentrations by Luminex multiplex array. Total protein concentration was analyzed by Bradford assay, and concentrations are expressed as $\mathrm{pg} / \mathrm{mg}$ total protein for each analyte. (A) In patients with TB, MMP-1 and MMP-3 levels were increased. MMP-13 (collagenase 3) was undetectable. (B) In contrast, levels of the inhibitors TIMP-1 and TIMP-2 were lower in patients with TB relative to those of controls. TIMP-3 and TIMP-4 were undetectable. Differences analyzed by Mann-Whitney $U$ test are shown. The box outline represents the 25th and 75th percentiles, the central line represents the median value, and the whiskers represent minimum and maximum values. Cont, control respiratory symptomatics.

that increased MMP concentrations measured by immunoassay were associated with greater proteolytic activity, demonstrating preserved potential for functional activation (Figure 2B). Chest radiographs for HIV-negative patients with TB were scored according to a standard classification of severity (10). MMP-1 concentrations correlated with degree of pulmonary inflammation and were 8.5 -fold higher in patients with more extensive lung disease than in patients with minor disease (Figure 2C; $P=0.030$ ).

$M$. tuberculosis selectively upregulates MMP-1 in buman monocytes. Next, we performed global analysis of all MMPs, TIMPs, and the related cell-surface a disintegrin and metallopeptidase proteins (ADAMs) with a functional protease domain in primary human monocytes to further profile the enzymes upregulated by M. tuberculosis. Infection increased mRNA levels of multiple MMPs at 24 hours (Figure $3 \mathrm{~A})$. MMP1 was both the most highly constitutively expressed collagenase by cycle threshold analysis (Figure 3A) and the most highly upregulated collagenase by infection (Figure $3 \mathrm{~B}$ ). In each donor studied, $M$. tuberculosis increased MMP1 gene expression 100 fold. No compensatory increase in gene expression of the inhibitors TIMP1TIMP4 or reversion-inducing cysteine-rich protein with Kazal motifs $(R E C K)$ was demonstrated. Relative gene expression of all other MMPs, TIMPs, and ADAMs is shown in Supplemental Figure 2.

Increased gene expression resulted in protease synthesis. MMP-1 secretion increased 46 fold at 24 hours after infection (Figure 4A; $P=<0.0001)$, and secretion of other MMPs closely reflected gene expression (Supplemental Figure 3). MMP-1 secretion was not increased by phagocytosis of latex beads but was increased by both UV-killed M. tuberculosis and the synthetic TLR-2 ligand $\mathrm{Pam}_{3} \mathrm{Cys}$ (Figure 4A), demonstrating that live mycobacteria were not required for MMP-1 upregulation and TLR-2 signaling increases MMP-1 secretion. M. tuberculosis infection increased monocyte migration through an extracellular matrix-coated transwell system (Figure 4B). Therefore, MMP upregulation results in a functional increase in matrix breakdown by infected cells. Finally,
Ro32-3555, a selective collagenase inhibitor of proven safety in humans (11), completely inhibited MMP-1 activity secreted by M. tuberculosis-infected primary human macrophages (Figure 4C).

Human MMP-1 expression in the mouse causes matrix destruction. Our human clinical and cellular studies implicate MMP-1 as the collagenase causing tissue destruction in TB, but animal modeling is necessary to confirm that MMP-1 degrades lung collagen in TB.
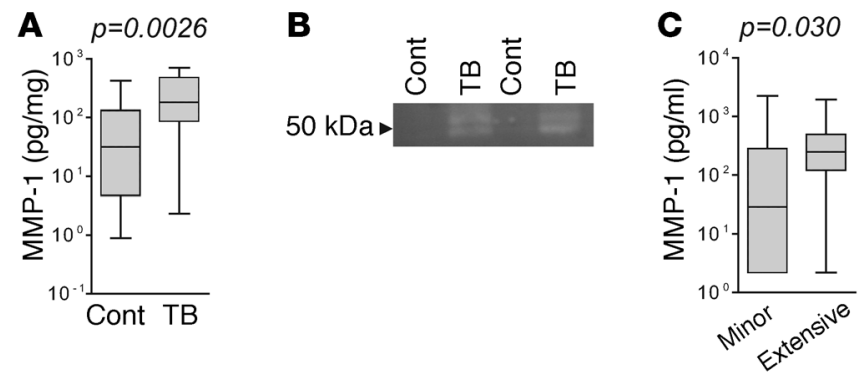

\section{Figure 2}

MMP-1 is increased in HIV-TB coinfection and is enzymatically active. (A) In HIV-positive patients, MMP-1 was increased in lung secretions from patients with TB compared with that in respiratory symptomatics. (B) Casein zymography. $20 \mu$ linduced sputum from respiratory symptomatics (Cont) or TB-infected patients (TB) was resolved on a casein zymogram and incubated in collagenase buffer at $37^{\circ} \mathrm{C}$ for 40 hours. After staining with Coomassie blue, induced sputum from patients with TB had greater caseinolytic activity, demonstrating that the increased MMP-1 detected by luminex array retains potential proteolytic activity. (C) Chest radiographs of patients with TB were scored for severity. MMP-1 concentrations in respiratory secretions were higher in patients with more extensive disease. Differences analyzed by Mann-Whitney $\mathrm{U}$ test are shown. For box-and-whisker plots, the box outline represents the 25th and 75th percentiles, the central line represents the median value, and the whiskers represent minimum and maximum values. 
A

MMP1

MMP2

MMP3

MMP4

MMP8

MMP9

MMP10

MMP11

MMP12

MMP13

MMP14

MMP15

MMP16

MMP17

MMP19

MMP20

MMP21

MMP23B

MMP24

MMP25

MMP26

MMP27

MMP28

Cont M. tuberculosis

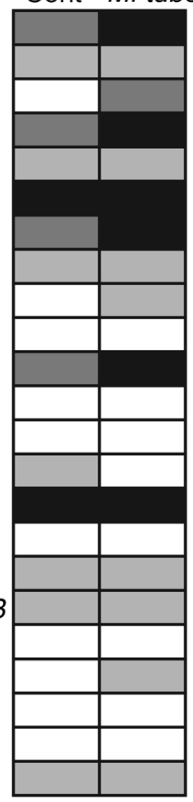

ADAM8

ADAM9

ADAM10

ADAM12

ADAM15

ADAM17

ADAM19

ADAM28

ADAM33

Cont M. tuberculosis

TIMP1

TIMP2

TIMP3

TIMP4

RECK
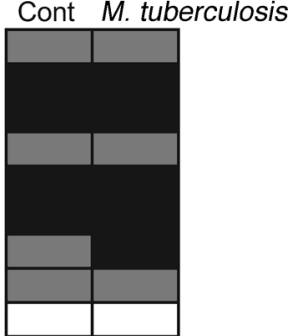

Cycle threshold

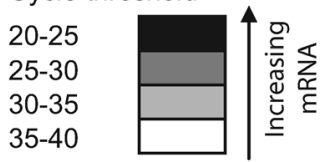

B

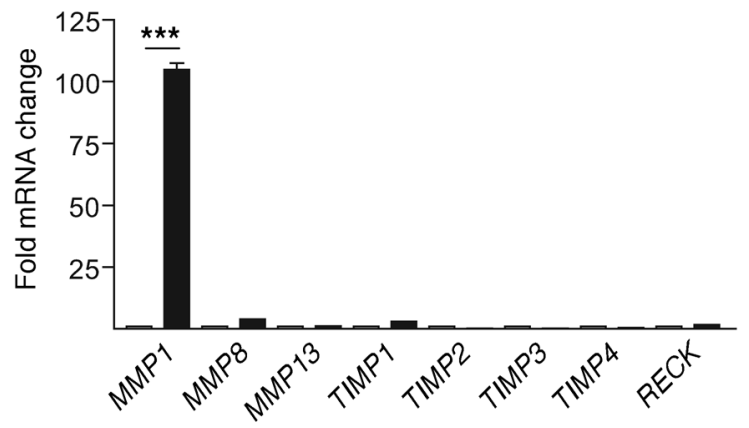

The mouse is a useful model of the immune response to $M$. tuberculosis, identifying key roles for $\mathrm{CD}^{+} \mathrm{T}$ cells and TNF- $\alpha, \mathrm{IL}-12$, and IFN- $\gamma$, which were subsequently confirmed in humans, but the mouse does not develop pathological changes similar to those of humans (4-6). The proposed mouse ortholog of MMP-1 is not expressed in the lung $(12,13)$ and lacks collagenolytic activity (12). Therefore, we infected mice that express human MMP-1 only in activated macrophages under control of the scavenger receptor A promoter/enhancer (14) with virulent $M$. tuberculosis at a low MOI to determine whether MMP-1 causes matrix destruction.

After 130 days of infection, the alveolar walls of M. tuberculosisinfected wild-type mice remained intact in areas of macrophage infiltration as shown by Masson's trichrome staining (Figure 5, $\mathrm{A}$ and $\mathrm{B})$. In contrast, in mice expressing human MMP-1, the alveolar walls were destroyed in areas of infection (Figure 5, C and D). In uninfected transgenic mice, no lung remodeling was demonstrated (Supplemental Figure 4). Alveolar damage within areas of infected lung was scored by a pathologist blinded to the mouse genotype, and a significant increase in tissue destruction was confirmed (Figure 5E). Minimal alveolar destruction occurred in wild-type mice compared with that in transgenic mice. After 4 and 7 months of infection, no difference in $M$. tuberculosis colony forming units was found between wild-type and MMP-1-expressing mice (Figure 5F and data not shown), demonstrating that increased matrix breakdown

\section{Figure 3}

M. tuberculosis selectively upregulates MMP1 gene expression. Primary human monocytes were infected with $M$. tuberculosis, and total RNA was extracted at 24 hours. (A) Gene expression profiling of all human MMPs, TIMPs, and ADAMs. Cycle threshold analysis for each gene is demonstrated, from white indicating low expression to black indicating high expression, and data are the mean of 3 donors infected on separate occasions. MMP1 is the collagenase most highly constitutively expressed. M. tuberculosis-infected cells upregulated MMP1, MMP3, MMP7, MMP8, MMP10, MMP12, and MMP14 compared with uninfected cells. Expression of $A D A M 17$ and $A D A M 19$ is also increased in infected monocytes. (B) M. tuberculosis upregulates MMP1 mRNA more potently than other secreted collagenases. MMP1 mRNA levels increased 100 fold in each donor 24 hours after infection. No compensatory increase in expression of inhibitors TIMP1-TIMP4 and RECK is demonstrated. Fold change in gene expression levels of all other MMPs, TIMPs, and ADAMs are shown in Supplemental Figure $2 .{ }^{* * *} P<0.001$.

was not the result of divergent mycobacterial proliferation. Tissue destruction was accompanied by increased MMP1 gene expression and enzyme concentrations in BALF and lung homogenate (Figure 5, G-I). In contrast to the MMP-1 upregulation, expression of mouse collagenases, MColA, MMP-8, and MMP-13, was not increased by M. tuberculosis infection in wild-type or transgenic mice (data not shown). McolA, the closest ortholog of human MMP-1, was not detected in the lung but was expressed in the testicular tissue, consistent with previous reports $(12,13)$. Virulent $M$. tuberculosis was necessary to drive tissue destruction, since neither lung immunopathology nor MMP-1 upregulation was demonstrated after infection with an attenuated strain of $M$. tuberculosis lacking RD1 (15), even when the initial infectious dose was 100-fold higher (data not shown). ZiehlNeelsen staining demonstrated acid-fast bacilli within infected macrophages in both wild-type and MMP-1-expressing mice (Figure 5, $\mathrm{J}$ and $\mathrm{K}$, and Supplemental Figure 5).

To quantify the effect of MMP-1 expression on matrix remodeling, lung sections were stained with Sirius red and imaged under polarized light. In wild-type mice, alveolar collagen was intact within areas of macrophage infiltration (Figure 6, A and C), while in transgenic mice collagen was degraded (Figure 6, B and D). Digital image analysis showed significantly reduced total collagen in MMP-1-expressing mice compared with that in wild-type mice (Figure 6E, $P=0.0073$ ). Elastin fibers were thinner and disorganized within areas of $M$. tuberculosis-driven inflammation as shown by Elastic-van Gieson staining, but no difference was observed between wild-type and MMP-1-expressing mice (data not shown). Finally, we studied type III collagen, the primary fibrillar collagen supporting the alveolar walls (7), which MMP-1 cleaves relatively specifically. In wild-type mice, type III collagen remained intact in the alveolar walls, while in MMP-1-expressing mice, it was degraded (Figure 6, F and G). To determine whether MMP-1 modulated cellular recruitment to the granuloma, total leukocytes, macrophages, and $\mathrm{CD} 4^{+} \mathrm{T}$ cells were analyzed by immunohistochemistry. No difference in cellular recruitment was demonstrated between wild-type and transgenic mice (Supplemental Figure 6).

\section{Discussion}

Together, analysis of clinical samples, primary human monocytes, and transgenic mice demonstrates that MMP-1 causes lung matrix destruction in TB. Collagen breakdown occurred in the absence of caseous necrosis, demonstrating that these are separate processes. 
A

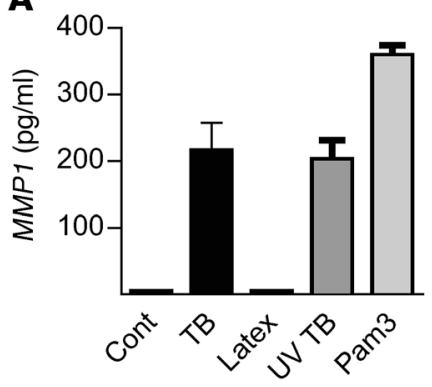

B

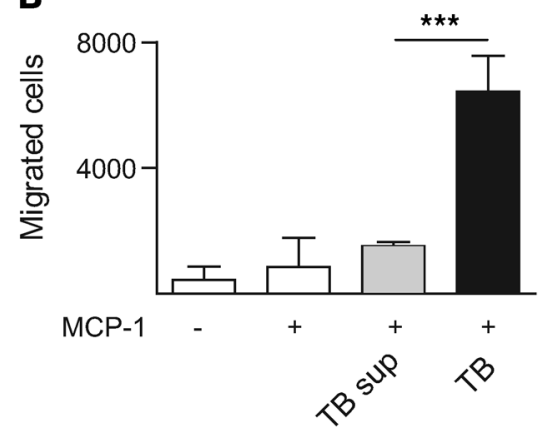

C

Std Cont TB

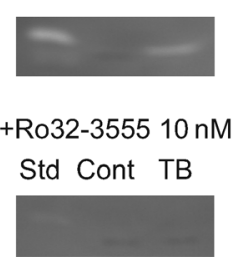

\section{Figure 4}

M. tuberculosis upregulates MMP-1 secretion and activity, which is inhibited by Ro32-3555. (A) M. tuberculosis-upregulated MMP1 gene expression causes increased secretion. Infected monocytes secreted 46-fold more MMP1 at 24 hours than uninfected cells. Monocyte stimulation with $3 \mu \mathrm{m}$ latex beads did not upregulate MMP1 secretion, but stimulation with UV-killed TB and the synthetic TLR-2 ligand Pam ${ }_{3}$ Cys upregulated MMP1 equivalently to live M. tuberculosis. Secretion of other MMPs by infected monocytes is shown in Supplemental Figure 3. (B) M. tuberculosis infection, but not TB supernatant (TB sup), causes increased monocyte migration across a Matrigel-coated transwell system, demonstrating that increased MMP secretion causes greater extracellular matrix degradation. (C) MMP-1 activity secreted by M. tuberculosis-infected human macrophages is completely inhibited by $10 \mathrm{nM}$ Ro32-3555, analyzed by casein zymography. Std, human MMP-1 standard $5 \mathrm{ng}$. ${ }^{* * *} P<0.001$.

This observation is consistent with postmortem studies in which TB cavity formation starts in areas of lipoid pneumonia, not in well-formed caseating granulomas (16). We found no difference in TNF- $\alpha$ between respiratory symptomatics and patients, consistent with previous reports (17), and demonstrated no difference in IFN- $\gamma$ protein levels, although others have found increased mRNA levels $(17,18)$. This divergence may result from other studies using healthy individuals as controls. The increase in MMP-1 concentrations in different patient cohorts, while cytokine concentrations were unchanged, supports the hypothesis that MMP-1 is a final effector of matrix destruction in TB.

MMP activity has previously been implicated in the immunopathology of TB, but a comprehensive analysis of MMPs in human TB has never been performed. Consistent with our findings, increased collagenase secretion by guinea pig macrophages stimulated by mycobacterial extracts was described 30 years ago (19). MMP1 gene expression is upregulated in primary human monocytes stimulated by mycobacterial lipoarabinomannan (20). In primary human macrophages, $M$. tuberculosis upregulates MMP-1 more potently than the vaccine strain BCG (21). More recently, gene expression profiling of macrophages demonstrated that MMP1 was the most potently upregulated gene in patients who developed pulmonary TB compared with those with latent disease (22). Similarly, microarray profiling of human TB granulomas has shown a 606-fold upregulation of MMP-1 expression compared with that in uninfected lung (23), and profiling of macaque lung tissue has demonstrated that $M M P 1$ is one of the most upregulated genes of the 1,584 induced by TB (24). In the zebrafish model, host transcriptome analysis comparing virulent and attenuated strains of Mycobacterium marinum found that although most genes were equally modulated by infection, the virulent bacterial strain upregulated 2 collagenases more potently than the attenuated strain (25). However, despite the accumulating evidence from whole-genome profiling implicating MMP-1 in matrix destruction, the significance seems to have been overlooked.

Since the extracellular matrix has numerous components degraded by different proteases, multiple MMPs may be involved in M. tuberculosis-driven immunopathology. Consistent with this, infection of MMP-9-deficient mice results in poor macrophage recruit- ment and granuloma formation (26). Recently, MMP-9 has been shown to regulate macrophage recruitment to $M$. marinum granulomas in the zebrafish model (27). However, MMP-9 is unable to cleave fibrillar collagens (8) and so cannot cause collagen breakdown that must precede pulmonary cavitation. Similarly, serine proteases, which have recently been implicated in control of TB (28), cannot degrade collagen. We demonstrated that monocytes expressed several MMPs constitutively, consistent with previous reports (29), but that $M$. tuberculosis infection consistently upregulated MMP-1 expression 100 fold. MMP-1 and MMP-3 were similarly upregulated in both patients with TB and M. tuberculosis-infected primary human monocytes. MMP-3 activates MMP-1 (8), therefore $M$. tuberculosis may initiate a proteolytic cascade resulting in tissue destruction. MMPs act in the pericellular environment (30), and so, although total MMP-1 is increased in respiratory secretions, net proteolytic activity distant from the site of disease may be unchanged due to an excess of constitutively expressed inhibitors.

Our data using what we believe to be a novel murine model of TB demonstrates a functional role for MMP-1 driving extracellular matrix remodeling in TB in the absence of caseous necrosis. The relative role of MMP-1 will be underestimated in this model, since stromal cell MMP secretion driven by M. tuberculosis will be absent $(27,31,32)$. The lack of a functional ortholog of MMP-1 in mice would explain why infection of immunodeficient mice can result in extremely high bacterial loads and necrosis but cavitation does not occur $(4,5)$. In immunodeficient mice that develop well-organized granulomas containing very high bacterial loads and necrosis, lesions have a fibrotic capsule (33), further suggesting that necrosis and matrix destruction are separate processes. Animals that cavitate after infection with $M$. tuberculosis, such as humans, primates, and rabbits (5), all have a preserved functional ortholog of MMP-1. In human disease, granulomas contain very few mycobacteria and yet extensive immunopathology occurs. Similarly, in MMP-1-expressing mice, paucibacillary disease without extracellular growth drives matrix destruction. MMP-1 expression did not modulate cellular recruitment to the granuloma, demonstrating that it directly cleaves matrix, consistent with its original function (34), as opposed to acting as a modulator of immune responses, which has been proposed for MMP-9 (35). We propose 

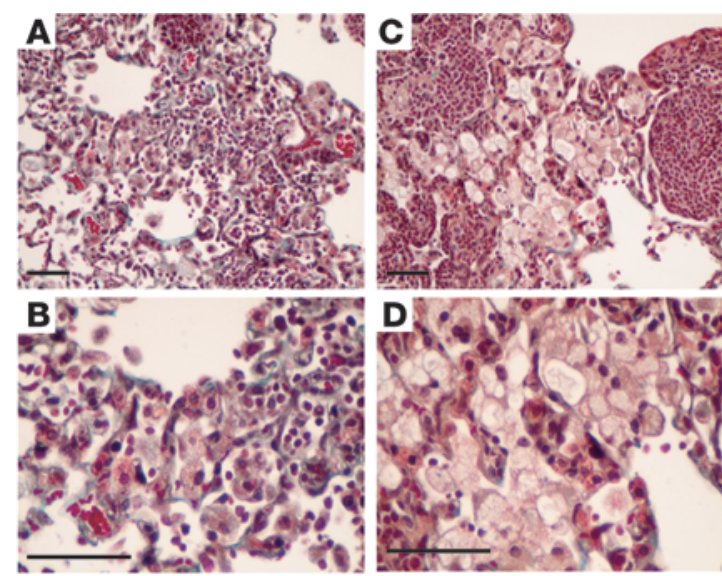

G

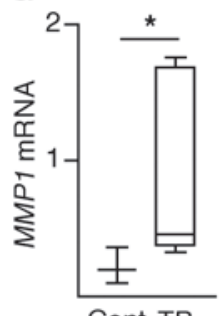

H

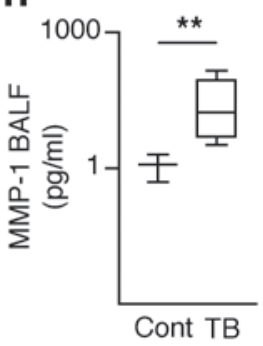

E

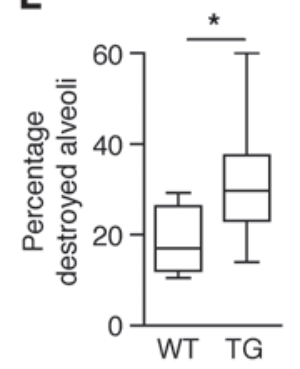

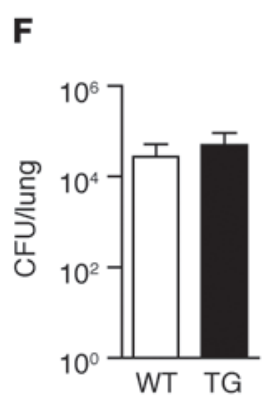

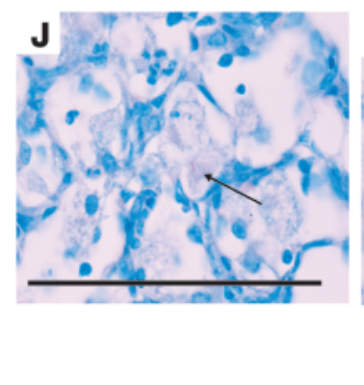

I

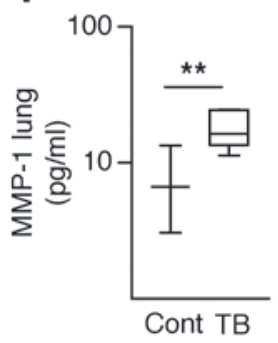

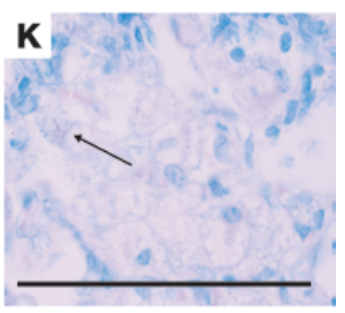

\section{Figure 5}

MMP-1 drives matrix destruction in TB granulomas of transgenic mice. Mice expressing human MMP-1 in activated macrophages and wild-type mice littermates were infected with $M$. tuberculosis H37Rv. At 130 days, mice were sacrificed. (A and $\mathbf{B}$ ) In wild-type mice, alveolar walls are intact on Masson's Trichrome staining. (C and D) In contrast, in MMP-1-expressing mice, alveolar walls have been destroyed within areas of pneumonia. (E) Alveolar wall integrity in regions of pneumonia was scored by a pathologist blinded to the mouse genotype. Increased alveolar wall destruction was demonstrated in mice expressing MMP-1. (F) No significant difference in colony counts was demonstrated at 230 days after infection. (G) Relative MMP1 mRNA levels are increased in infected transgenic mice. (H) MMP-1 protein concentration is increased in BALF and (I) lung homogenates of $M$. tuberculosis-infected mice compared with those of uninfected transgenic mice. ( $\mathbf{J}$ and $\mathbf{K})$ Acid-fast bacilli are demonstrated in infected macrophages in ( $\mathbf{J}$ ) wild-type and (K) MMP-1-expressing mice. High resolution images are shown in Supplemental Figure 5. For each experiment, a minimum of 4 mice per group were studied, and a total of 4 separate infection experiments were performed. For box-and-whisker plots, the box outline represents the 25th and 75th percentiles, the central line represents the median value, and the whiskers represent minimum and maximum values. ${ }^{\star} P<0.05,{ }^{* \star} P<0.01$ by Student's $t$ test. Scale bars: $50 \mu \mathrm{m}$ for all panels.

that the final effector of cavitation in TB is excess collagenase activity, while any imbalance of the adaptive immune response that has been postulated to drive cavitation must be upstream of MMP activity $(2-4,6,36)$.

Targeting MMP-1 activity may reduce the pathology that results in the morbidity and mortality of TB. P-amino-salicylic acid, used to treat TB for 60 years but with a poorly defined mechanism of action, inhibits MMP-1 secretion by M. tuberculosis-infected macrophages (37), suggesting that an established treatment for TB may act by limiting tissue destruction. We demonstrate that Ro32-3555, a compound that has been used in phase III clinical trials for arthritis (11), can suppress M. tuberculosis-driven MMP-1 activity. MMP-1 causes matrix destruction in $\mathrm{TB}$, and therefore we believe it represents a novel therapeutic target to limit immunopathology.

\section{Methods}

Induced sputum and BALF collection and analysis. The study was approved by the Royal Free Hospital Ethics committee (LREC6236). Written informed consent was obtained from patients being investigated for possible TB. In the initial study, all patients studied were HIV negative, and a second study was performed in patients with confirmed HIV infection. Induced sputum and BALF was obtained, processed as previously described (38), and frozen at minus $20^{\circ} \mathrm{C}$. Samples were sterile filtered through a $0.2-\mu \mathrm{m}$ membrane (Millipore) and analyzed on the Luminex 200 platform. Luminex beads for MMPs and TIMPs (R\&D Systems) and for cytokines (Bio-Rad) were analyzed as per manufacturer's instructions. Total protein in each sample was analyzed by Bradford assay (Bio-Rad). Statistical analysis was performed with STATA 10 (Statacorp LP).

Monocyte purification and infection. PBMCs were isolated from single donor buffy coats (National Blood Transfusion Service, United Kingdom) by density centrifugation (Amersham Biosciences) and resuspended in RPMI, and monocytes were purified by adhesion for 1 hour. The monolayer was washed 6 times with HBSS to remove nonadherent cells and then infected with M. tuberculosis H37Rv at an MOI of 1 or M. tuberculosis killed by UV irradiation for 1 hour, $3 \mu \mathrm{m}$ latex beads (Sigma-Aldrich), and the synthetic TLR-2 ligand Pam ${ }_{3}$ Cys at $5 \mu \mathrm{g} / \mathrm{ml}$ (Invivogen). Gene expression and secretion was analyzed at 24 hours. For macrophage experiments, monocytes were matured for 4 days in $100 \mathrm{ng} / \mathrm{ml} \mathrm{M-CSF}$ as previously described (21). MMP secretion was analyzed 3 days after $M$. tuberculosis infection by Luminex array.

Gene expression analysis by real-time PCR. Monocytes were lysed using TriReagent (Sigma-Aldrich), and total RNA was extracted. $1 \mu \mathrm{g}$ RNA was reverse transcribed using $2 \mu \mathrm{g}$ random hexamers (Amersham Biosciences) and 200 

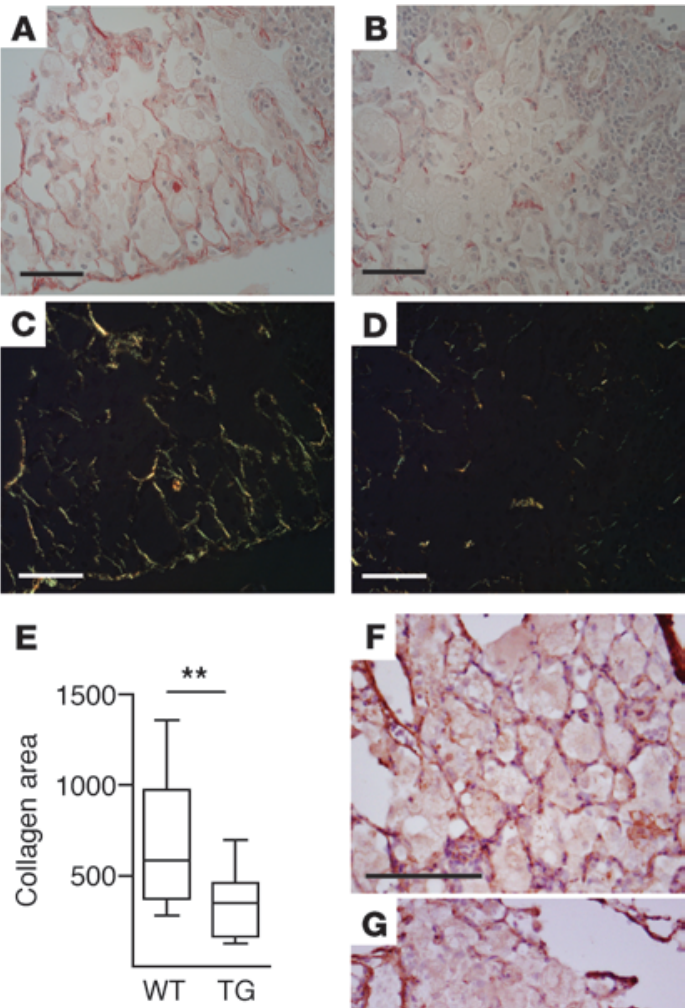

units of SuperScript II reverse transcriptase (Invitrogen), according to the supplier's instructions. qPCR reactions were done on the ABI Prism 7700 (Applied Biosystems) according to previously described methods (21). The cycle threshold at which amplification entered the exponential phase was determined, and this number was used to indicate the amount of target RNA in each sample.

Cell migration assay. Assays were performed in 24-well plates with $8-\mu \mathrm{m}$ transwell inserts (Nunc). Membranes were precoated with $1 \mathrm{mg}$ Matrigel (BD Biosciences) for 1 hour at $37^{\circ} \mathrm{C}$. Freshly isolated PBMCs $\left(1 \times 10^{6}\right.$ cells in $300 \mu \mathrm{RPMI}$ ) were seeded in the upper compartment, and the lower compartment was filled with RPMI containing $100 \mathrm{ng} / \mathrm{ml} \mathrm{MCP-1} \mathrm{(Pepro-}$ Tech). Cells were infected with $M$. tuberculosis at MOI of 1 and incubated overnight at $37^{\circ} \mathrm{C}$. Cells were fixed in $2 \%$ paraformaldehyde and counted by flow cytometry (BD FACSCalibur).

Casein zymography. For assessment of MMP-1 activity, samples were analyzed on $0.05 \%$ casein gels (Invitrogen) and incubated for 40 hours in collagenase buffer at $37^{\circ} \mathrm{C}$ as previously described (21). Caseinolytic activity was revealed by Coomassie blue staining (Pharmacia). Inhibition assays were performed by adding $10 \mathrm{nM}$ Ro32-3555 (Tocris Bioscience) to collagenase buffer prior to incubation.

Mouse infection and analysis. All mouse experiments were approved by the United Kingdom Home Office and performed in accordance with the appropriate project licence. Ten- to twelve-week-old C57BL/6 mice expressing human MMP-1 (14) and their wild-type littermates were infected with 400 colony forming units $M$. tuberculosis H37Rv intratracheally (minimum 4 mice per group) or an attenuated $\triangle \mathrm{RD} 1 \Delta$ panCD M. tuberculosis strain, provided by Michelle Larsen and Bill Jacobs, Albert Einstein College of Medicine, New York, New York, USA. Noninfected controls were injected

\section{Figure 6}

M. tuberculosis-driven MMP-1 expression causes collagen destruction. (A and $\mathbf{B})$ Total collagen was stained with Sirius red and ( $\mathbf{C}$ and D) imaged under polarized light. (A and $\mathbf{C}$ ) In wild-type mice, collagen is intact in areas of macrophage infiltration, (B and $\mathbf{D})$ but in mice expressing MMP-1, collagen is degraded. (E) Collagen is reduced in infected lungs of MMP-1-expressing mice, by digital image analysis of birefringence. The box outline represents the 25th and 75th percentiles, the central line represents the median value, and the whiskers represent minimum and maximum values. ${ }^{* *} P<0.01$ by Student's $t$ test. ( $\mathbf{F}$ and $\mathbf{G}$ ) Type III collagen is degraded in areas of macrophage infiltration. (F) In wild-type mice, type III alveolar wall collagen is intact, (G) while in transgenic mice, type III collagen has been degraded. Scale bars: $50 \mu \mathrm{m}$ for all panels.

intratracheally with PBS. Three mice were sacrificed on day 1 to confirm the infectious dose. Experimental mice were sacrificed by terminal overdose of anaesthetic at either 130 days ( 3 experiments) or 230 days (1 experiment) after infection. An intravenous cannula was inserted in the trachea and sutured into place, and a bronchoalveolar lavage was performed with $1 \mathrm{ml}$ sterile PBS. The left lung was clamped off and divided in 2 . Half was placed in RNAlater (Qiagen) for mRNA analysis, and half was placed in PBS for protein analysis and colony counting. The right lung was then inflated with formalin under $12 \mathrm{~cm}$ pressure for 5 minutes and then immersed in formalin. After 24 hours, the right lung was embedded in paraffin, and serial 3- $\mu \mathrm{m}$ sections were taken of the whole lung. Hematoxylin and eosin, Masson's trichrome, Picrosirius red, Ziehl-Neelsen, Elastic-van Gieson, and type III collagen immunohistochemistry (primary antibody, Abcam rabbit polyclonal anti-collagen III) stains were performed. To quantify alveolar wall integrity, slides were randomized, and the integrity of each alveolus within areas of macrophage infiltration was scored by a pathologist blinded to the genotype of the mice. Results were expressed as the percentage of destroyed alveoli within the area of inflammation analyzed. For quantitation of total collagen, Picrosirius-stained sections were imaged under birefringence (Leica DM2500), 4 digital images per mouse were captured, and the area of collagen was determined by Leica Qwim software.

For mRNA analysis, the lung was placed in $1 \mathrm{ml} \mathrm{TRIzOL}$ (Invitrogen) and disrupted by a rotating blade homogenizer (Omni International). Total mRNA was extracted and MMP1 gene expression was analyzed as above. For MMP-1 protein analysis and colony count determination, the lung was placed in $1 \mathrm{ml}$ sterile PBS and dissociated by a rotating blade homogenizer. Colony counting was performed by plating serial 1:10 dilutions of lung homogenate on 7H11 agar (BD Biosciences), and colonies were counted at 3 weeks. Lung homogenate and BALF was sterile filtered through a $0.2-\mu \mathrm{m}$ filter (Millipore). MMP-1 and cytokine concentrations were analyzed by Luminex bead array as above.

To analyze cellular recruitment to TB granulomas, formalin-fixed, paraffin-embedded sections were stained for total leukocytes with anti-CD45 (Leukocyte Common Antigen, BD Pharmingen), and macrophages were stained with F4/80 antibody (Abcam), with antigen retrieval of $0.1 \%$ tryp$\sin$ for 120 minutes. $\mathrm{CD} 4^{+}$cells were analyzed on adjacent sections with anti-CD4 (Abcam), with antigen retrieval of $3 \mathrm{M} \mathrm{HCl}$ for 20 minutes and then autoclaving with $\mathrm{pH} 6.0$ citrate buffer for 20 minutes. Total cells for each granuloma were counted by a pathologist blinded to the genotype of the mice, and data are expressed as the percentage of macrophages or $\mathrm{CD} 4^{+}$ cells of total granuloma inflammatory cells.

Statistics. Statistical analysis was performed with STATA 10.0 (StataCorp). Clinical data were analyzed by Mann-Whitney U test, while cellular data were analyzed by 1 -tailed $t$ test. A $P$ value of $<0.05$ was considered significant. For all bar graphs, the mean $\pm \mathrm{SD}$ is plotted. 


\section{Acknowledgments}

This work was supported by the United Kingdom National Institute for Health Research (NIHR) and the Scadding Morriston Davies Travel Fellowship (to P. Elkington) and NIH RO1HL086936 (to J. D'Armiento). P. Elkington and J.S. Friedland are grateful for support from the NIHR Biomedical Research Centre funding scheme at Imperial College. We thank Sian Floyd for reviewing statistical analyses and Tina Zelonina, Mahrokh Nohadani, Tomoe Shiomi, and Daniel Wells for excellent technical assistance.
Received for publication November 4, 2010, and accepted in revised form February 9, 2011.

Address correspondence to: Paul Elkington, Department of Infectious Diseases and Immunity, Imperial College London, Hammersmith Campus, Du Cane Road, London W12 0NN, United Kingdom. Phone: 00.44.20.8383. 2733; Fax: 00.44.20.8383.3394; E-mail: p.elkington@ imperial.ac.uk.
1. Dye C, Williams BG. The population dynamics and control of tuberculosis. Science. 2010; 328(5980):856-861.

2. Russell DG. Who puts the tubercle in tuberculosis? Nat Rev Microbiol. 2007;5(1):39-47.

3. Yoder MA, Lamichhane G, Bishai WR. Cavitary pulmonary tuberculosis: The Holey Grail of disease transmission. Current Sci. 2004;86(1):74-81.

4. North RJ, Jung YJ. Immunity to tuberculosis. Annu Rev Immunol. 2004;22:599-623.

5. Young D. Animal models of tuberculosis. Eur J Immunol. 2009;39(8):2011-2014.

6. Cooper AM. Cell-mediated immune responses in tuberculosis. Annu Rev Immunol. 2009;27:393-422.

7. Davidson JM. Biochemistry and turnover of lung interstitium. Eur Respir J. 1990;3(9):1048-1063.

8. Greenlee KJ, Werb Z, Kheradmand F. Matrix metalloproteinases in lung: multiple, multifarious, and multifaceted. Physiol Rev. 2007;87(1):69-98.

9. Page-McCaw A, Ewald AJ, Werb Z. Matrix metalloproteinases and the regulation of tissue remodelling. Nat Rev Mol Cell Biol. 2007;8(3):221-233.

10. Falk A, O'Connor JB, Pratt PC. Classification of pulmonary tuberculosis. In: Falk A, O'Connor JB, Pratt PC, Webb JA, Wier JA, Wolinsky E, eds. Diagnosis Standards and Classification of Tuberculosis. New York, New York, USA: National Tuberculosis and Respiratory Disease Association; 1969:68-76.

11. Hemmings FJ, Farhan M, Rowland J, Banken L, Jain R. Tolerability and pharmacokinetics of the collagenase-selective inhibitor Trocade in patients with rheumatoid arthritis. Rheumatology (Oxford). 2001;40(5):537-543.

12. Balbin $M$, et al. Identification and enzymatic characterization of two diverging murine counterparts of human interstitial collagenase (MMP-1) expressed at sites of embryo implantation. J Biol Chem. 2001;276(13):10253-10262.

13. Nuttall RK, Sampieri CL, Pennington CJ, Gill SE, Schultz GA, Edwards DR. Expression analysis of the entire MMP and TIMP gene families during mouse tissue development. FEBS Lett. 2004;563(1-3):129-134.

14. Lemaitre V, O'Byrne TK, Borczuk AC, Okada Y, Tall AR, D'Armiento J. ApoE knockout mice expressing human matrix metalloproteinase- 1 in macrophages have less advanced atherosclerosis. J Clin Invest. 2001;107(10):1227-1234.

15. Sambandamurthy VK, et al. Mycobacterium tuberculosis DeltaRD1 DeltapanCD: a safe and limited replicating mutant strain that protects immunocompetent and immunocompromised mice against experimental tuberculosis. Vaccine. 2006;24(37-39):6309-6320.

16. Hunter RL, Jagannath C, Actor JK. Pathology of postprimary tuberculosis in humans and mice: contradiction of long-held beliefs. Tuberculosis (Edinb). 2007;87(4):267-278.

17. Taha RA, Kotsimbos TC, Song YL, Menzies D, Hamid Q. IFN-gamma and IL-12 are increased in active compared with inactive tuberculosis. Am J Respir Crit Care Med. 1997;155(3):1135-1139.

18. Almeida AS, et al. Tuberculosis is associated with a down-modulatory lung immune response that impairs Th1-type immunity. J Immunol. 2009;183(1):718-731.

19. Wahl SM, Wahl LM, McCarthy JB, Chedid L, Mergenhagen SE. Macrophage activation by mycobacterial water soluble compounds and synthetic muramyl dipeptide. J Immunol. 1979;122(6):2226-2231.

20. Chang JC, Wysocki A, Tchou-Wong KM, Moskowitz N, Zhang Y, Rom WN. Effect of Mycobacterium tuberculosis and its components on macrophages and the release of matrix metalloproteinases. Thorax. 1996;51(3):306-311.

21. Elkington PT, et al. Mycobacterium tuberculosis, but Not Vaccine BCG, Specifically Upregulates Matrix Metalloproteinase-1. Am J Respir Crit Care Med. 2005;172(12):1596-1604.

22. Thuong NT, et al. Identification of tuberculosis susceptibility genes with human macrophage gene expression profiles. PLoS Pathog. 2008; 4(12):e1000229.

23. Kim MJ, et al. Caseation of human tuberculosis granulomas correlates with elevated host lipid metabolism. EMBO Mol Med. 2010;2(7):258-274.

24. Mehra $S$, et al. Transcriptional reprogramming in nonhuman primate (rhesus macaque) tuberculosis granulomas. PLoS ONE. 2010;5(8):e12266.

25. van der Sar AM, Spaink HP, Zakrzewska A, Bitter W, Meijer AH. Specificity of the zebrafish host transcriptome response to acute and chronic mycobacterial infection and the role of innate and adaptive immune components. Mol Immunol. 2009; 46(11-12):2317-2332.

26. Taylor JL, et al. Role for matrix metalloproteinase 9 in granuloma formation during pulmonary Mycobacterium tuberculosis infection. Infect Immun. 2006;74(11):6135-6144.
27. Volkman HE, Pozos TC, Zheng J, Davis JM, Rawls JF, Ramakrishnan L. Tuberculous granuloma induction via interaction of a bacterial secreted protein with host epithelium. Science. 2010;327(5964):466-469.

28 . Reece ST, et al. Serine protease activity contributes to control of Mycobacterium tuberculosis in hypoxic lung granulomas in mice. J Clin Invest. 2010;120(9):3365-3376.

29. Welgus HG, et al. Neutral metalloproteinases produced by human mononuclear phagocytes. Enzyme profile, regulation, and expression during cellular development. J Clin Invest. 1990;86(5):1496-1502.

30. Murphy G, Nagase H. Localizing matrix metalloproteinase activities in the pericellular environment. FEBS J. 2011;278(1):2-15.

31. Elkington PT, et al. Mycobacterium tuberculosis Up-Regulates Matrix Metalloproteinase-1 Secretion from Human Airway Epithelial Cells via a p38 MAPK Switch. J Immunol. 2005;175(8):5333-5340.

32. O'Kane CM, Elkington PT, Friedland JS. Monocytedependent oncostatin M and TNF-alpha synergize to stimulate unopposed matrix metalloproteinase$1 / 3$ secretion from human lung fibroblasts in tuberculosis. Eur Immunol. 2008;38(5):1321-1330.

33. Pichugin AV, Yan BS, Sloutsky A, Kobzik L, Kramnik I. Dominant role of the sst 1 locus in pathogenesis of necrotizing lung granulomas during chronic tuberculosis infection and reactivation in genetically resistant hosts. Am J Pathol. 2009;174(6):2190-2201.

34. Brinckerhoff CE, Matrisian LM. Matrix metalloproteinases: a tail of a frog that became a prince. Nat Rev Mol Cell Biol. 2002;3(3):207-214.

35. Opdenakker G, Van den Steen PE, Van Damme J. Gelatinase B: a tuner and amplifier of immune functions. Trends Immunol. 2001;22(10):571-579.

36. Dannenberg AM Jr. Liquefaction and cavity formation in pulmonary TB: a simple method in rabbit skin to test inhibitors. Tuberculosis (Edinb). 2009;89(4):243-247.

37. Rand L, Green JA, Saraiva L, Friedland JS, Elkington PT. Matrix metalloproteinase- 1 is regulated in tuberculosis by a p38 MAPK-dependent, p-aminosalicylic acid-sensitive signaling cascade. J Immunol. 2009;182(9):5865-5872.

38. Breen RA, et al. Rapid diagnosis of smear-negative tuberculosis using immunology and microbiology with induced sputum in HIV-infected and uninfected individuals. PLoS One. 2007;2(12):e1335. 